\title{
Reflection of low-frequency equatorial waves at arbitrary western boundaries
}

\author{
by Mark A. Cane' and Peter R. Gent ${ }^{2}$
}

\begin{abstract}
Procedures are developed for calculating effects of western boundaries of arbitrary geometry on low-frequency equatorial waves. We show that the mass flux incident at the coast is all returned eastward by equatorial Kelvin waves. Short Rossby waves generated as part of the reflection serve only to redistribute mass along the coast. For the case of a uniformly sloping coast we present formulae and graphical results for the amplitude of the equatorial Kelvin wave reflected at the coast due to incident free Rossby waves. Results are shown to depend on the single parameter $\gamma \omega$, where $\gamma$ is the slope of the coast and $\omega$ is the nondimensional frequency. Similar results are presented for a special class of forced problems. Possible implications for equatorial currents in the Indian Ocean are discussed.
\end{abstract}

\section{Introduction}

Beginning with the seminal works of Lighthill (1969) and Moore (1968) the theory of equatorial waves has undergone extensive development and has been successfully applied to a wide variety of problems. (Cane and Sarachik, 1983, reviews the recent literature.) Some of the most interesting and important of this work concerns the reflection of zonally propagating waves at coasts, but in almost all cases the coastline is assumed to lie along a meridian, normal to the direction of propagation.

An important exception is Lighthill (1969), which considers the reflection of low-frequency waves at the sloping western boundary of the Indian Ocean. However, Lighthill's treatment is in error because of its neglect of the equatorial Kelvin wave, which typically plays a significant role in the reflection process. The present work considers a more general form of the same problem: the reflection of low-frequency waves at arbitrary western boundaries. In fact, our approach to the problem is rather similar to Lighthill's. It builds on the theory developed in a series of papers by Cane and Sarachik (most relevant here are Cane and Sarachik, 1977 and 1981, henceforth CSII and CSIV) and may properly be considered a generalization of that theory.

The plan of the paper is as follows. The next section presents the theory and develops formulae for the relevant reflection coefficients. The following sections present results

1. Center for Meteorology and Physical Oceanography, Massachusetts Institute of Technology, Cambridge, Massachusetts, 02139, U.S.A.

2. National Center for Atmospheric Research, P.O. Box 3000, Boulder, Colorado, 80307, U.S.A. 
for individual equatorial waves, for the sum of a Kelvin wave and its eastern boundary reflection (Cane and Moore, 1981), a combination of particular importance in forced problems, and for a class of wind-forced problems previously considered in CSIV and in Gent et al. (1983). The final section summarizes our results and discusses their possible significance.

\section{Method of solution}

We will consider periodic motions in a linear shallow water system on an equatorial beta plane (e.g., CSIV). At low frequencies ( $\omega \ll 1$, where $\omega$ is the nondimensional frequency), four types of wave motions are possible:

(i) long eastward propagating equatorial Kelvin waves,

(ii) westward propagating long Rossby waves,

(iii) eastward propagating short Rossby waves,

(iv) westward propagating long coastal Kelvin waves, trapped to zonally oriented coasts.

[Obviously type (iv) waves only exist in a meridionally bounded basin. See Cane and Sarachik (1979) for an extended discussion.] All of these modes are meridionally standing, propagating only zonally. The directions indicated are the directions which energy propagates. Hence the reflection problem amounts to specifying the amplitudes of the long Rossby waves (ii) and calculating the reflection coefficients of wave types (i) and (iii). The appropriate boundary condition for the problem is that

$$
\mathbf{u} \cdot \mathbf{n}=\mathbf{0}
$$

at every point along the coast, where $\mathbf{u}$ is the velocity and $\mathbf{n}$ is the unit normal to the coastline.

First consider the short Rossby waves (iii). As has been argued elsewhere (e.g., CSIV, p. 656ff), their group velocity is so small $\left[0\left(\omega^{2}\right)\right]$ that even a small amount of friction traps them to the western boundary, where they form a western boundary current. Further, each of these waves satisfies the dispersion relation

$$
k=-\omega^{-1}+0(\omega) \text {. }
$$

It is then easy to show that each is nondivergent to $0\left(\omega^{2}\right)$, so there exists a streamfunction

$$
\phi(x, y, \mathrm{t})=\tilde{\phi}(y) \exp i[\omega t+x / \omega]
$$

such that

$$
\mathbf{u}^{B}=\mathbf{k} \times \nabla \phi
$$

where $\mathbf{u}^{B}$ is the velocity of the sum of these short waves. 
The problem may now be rephrased: given a wave of frequency $\omega$ incident at the coast, determine the function $\phi$ at the coast and the amplitude $A_{K}$ of the reflected Kelvin wave. Let the coast extend from a latitude $Y_{S}$ to a latitude $Y_{N}$ and let $s$ denote distance along the coast. Define $s_{N}=s\left(Y_{N}\right)$ and $s_{S}=s\left(Y_{S}\right)$. The net mass flux normal to the coast attributable to the sum of short waves is

$$
U^{B}=\int_{s_{s}}^{s_{N}} \mathbf{u}^{B} \cdot \mathbf{n} d s=-\int_{s_{s}}^{s_{N}} \frac{\partial \phi}{\partial s} d s=\phi\left(Y_{S}\right)-\phi\left(Y_{N}\right) .
$$

If the latitudes $Y_{S}$ and $Y_{N}$ are finite, then we will also assume that the northern and southern boundaries at those latitudes are zonally oriented. It then follows that the meridional velocity $\partial \phi / \partial x=0$ and hence that both $\phi\left(Y_{S}\right)$ and $\phi\left(Y_{N}\right)$ are constant (independent of $x$ ). Since the boundary flow $\mathbf{u}^{8}$ vanishes for all $y$ as $x \rightarrow \infty$ it must be the case that $\phi\left(Y_{S}\right)=\phi\left(Y_{N}\right){ }^{3}$ We conclude that the normal mass flux $U^{B}$ is also zero. If the latitudes $Y_{S}$ and $Y_{N}$ are taken to be infinite then all components of the sum composing $\phi$ approach zero as $|y| \rightarrow \infty$, so $\phi$ also goes to zero at infinity and it again follows that $U^{B}=0$.

The last argument also requires that the sum of short Rossby waves be uniformly convergent. (To appreciate the potential difficulty, recall that the sum of Rossby modes that makes up the reflection of a Kelvin wave at an eastern boundary has a nonzero height field for all $y$ in the long wave approximation; see CSII p. 406.) We may establish that the sum does indeed go to zero at high latitudes as follows. Moore (1968) showed that at high latitudes the energy flux associated with these boundary modes is equatorward, a principle that will hold as well for sloping coasts. This is the basis for Moore's algorithm (see Moore and Philander, 1977) demonstrating that at a western boundary the meridional index number of all reflected waves is less than or equal to the index number of the incoming waves. Hence if the incident waves go to zero at all latitudes $|y|>L$, so will the reflected waves. The same conclusion is evident from the boundary condition (1) when one adds the consideration that the only other contributor to $\mathbf{u} \cdot \mathbf{n}$ at the boundary, namely the equatorial Kelvin wave, is exponentially small at latitudes $|y|>L \gg 1$.

We conclude that any sum of short Rossby waves has zero mass flux normal to the coast, and merely redistributes mass along the coast. Therefore, for all western coastline geometries the incoming mass flux normal to the coast due to all the long Rossby waves is balanced by the outgoing mass flux associated with the Kelvin wave. This important result is a generalization of that for straight north-south coasts found in CSII.

The principle stated in the preceding paragraph may be used to calculate the amplitude $A_{K}$ of the reflected Kelvin wave in terms of the incident long waves without considering the short waves at all. Let $U_{K}$ be the normal mass flux for a Kelvin wave of unit amplitude and let $U_{I}$ be the normal mass flux due to the incident long waves.

3. For the form (2) $\phi \rightarrow 0$ as $x \rightarrow \infty$ with nonzero friction ( $\omega$ complex). 


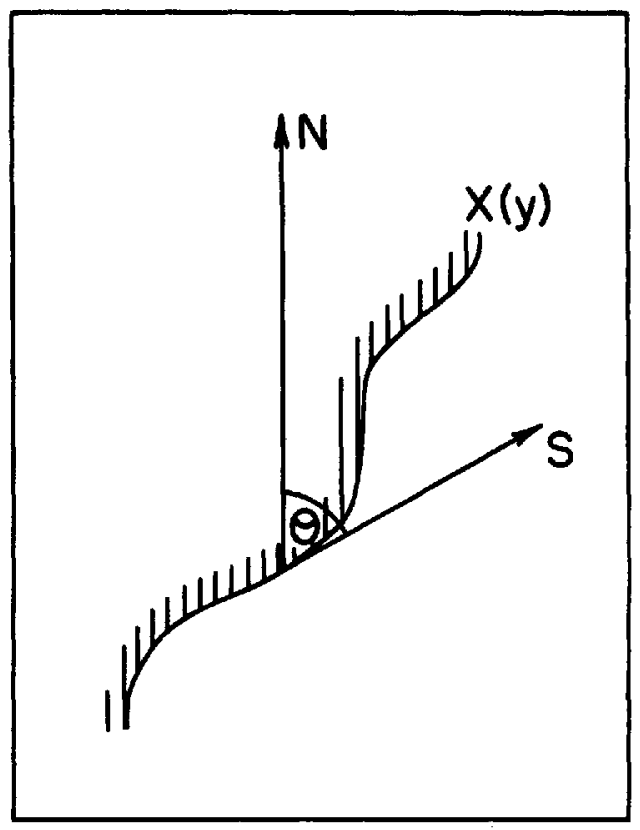

Figure 1. The geometry of the coastline.

Then

$$
A_{K}=-U_{I} / U_{K} .
$$

Once $A_{K}$ is known the boundary current may be found directly from (1), (2), and (3).

Useful computational formulae for the terms on the right-hand side of (4) may be found as follows. Let $u$ and $v$ denote the velocity components in the $x$ (eastward) and $y$ (northward) directions, respectively. Let the coastal geometry be defined as in Figure 1; i.e., let the curve $x=X(y)$ define the coastline and let $\theta$ be the angle that the coastline makes with north. Define

$$
\gamma=\tan \theta=d X / d y .
$$

Again denoting distance along the coast by $s$, the mass flux normal to the coast associated with $\mathbf{u}=(u, v)$ is

$$
\begin{aligned}
& U=\int_{s_{S}}^{s_{N}} \mathbf{u} \cdot \mathbf{n} d s=\int_{Y_{s}}^{Y_{N}}(u \cos \theta-v \sin \theta) d y / \cos \theta \\
& U=\int_{Y_{s}}^{Y_{N}}[u(X(y), y)-\gamma(y) v(X(y), y)] d y .
\end{aligned}
$$

We have written the arguments explicitly to emphasize that all terms are functions of $y$ only. However, (6) is not valid if $\theta=90^{\circ}$, so that $\gamma$ is infinite and $X(y)$ may not be single-valued. This case occurs if part of the western boundary becomes zonally 
oriented; we exclude this possibility here. (This case is considered in Du Penhoat et al., 1983.)

\section{Results for straight coasts: reflection of free Rossby waves}

We now specialize to the case of a straight coast; that is, $\gamma$ is a constant. Without loss of generality we may then take $X(y)=\gamma y$. We will also take the coastline to extend from $-\infty$ to $+\infty$, gaining simplicity and analytic tractability. As long as the incident motion is confined to a finite region of constant slope the extension to infinity introduces no appreciable error. This is a consequence of the fact, noted above, that the reflected motions are more equatorially confined than the incident ones. (More precisely, the long wave theory is good to $0\left(\omega^{2}\right)$ and the equatorial waves decay exponentially as $y \rightarrow \infty$. If the extension misrepresents the flow only poleward of a latitude where the amplitudes of these waves are only $0\left(\omega^{2}\right)$, then the order of accuracy of the theory is unchanged.)

A single equatorial wave at frequency $\omega$ has the form

$$
(u(x, y, t), v(x, y, t))=\exp i(\omega t-k x)(u(y), v(y)) .
$$

Dropping explicit reference to the time dependence, substituting this form in (6), and assuming a straight, infinite coast yields

$$
U=\int_{-\infty}^{+\infty}[u(y)-\gamma v(y)] e^{-i k \gamma y} d y,
$$

which shows that $U$ is essentially the Fourier transform of $(u-\gamma v)$ with argument $k \gamma$. For equatorial waves we need only the Fourier transforms of the Hermite functions $\psi_{m}(y)$ (see, for example, the appendix to CSIV for definitions of these and other equatorial wave quantities). The necessary transform is standard:

$$
\int_{-\infty}^{+\infty} \psi_{m}(y) e^{i l y} d y=\sqrt{2 \pi}(i)^{m} \psi_{m}(l)
$$

For the Kelvin wave, $k=\omega$,

$$
u_{K}=h_{K}=\frac{\psi_{0}(y)}{\sqrt{2}}, \quad v_{K}=0,
$$

so the integrated normal mass flux of a unit amplitude Kelvin wave is

$$
U_{K}=\sqrt{\pi} \psi_{0}[\gamma \omega]
$$

The long Rossby waves have the form $\exp i(\omega t-k x)\left[u_{m}, v_{m}, h_{m}\right]$ with $k=$ $-(2 m+1) \omega$ and

$$
\begin{aligned}
\left(u_{m}, v_{m}, h_{m}\right)= & \left\{2^{-3 / 2}\left[(m+1)^{-1 / 2} \psi_{m+1}-m^{-1 / 2} \psi_{m-1}\right], i \omega \psi_{m},\right. \\
& \left.2^{-3 / 2}\left[(m+1)^{-1 / 2} \psi_{m+1}+m^{-1 / 2} \psi_{m-1}\right]\right\} .
\end{aligned}
$$



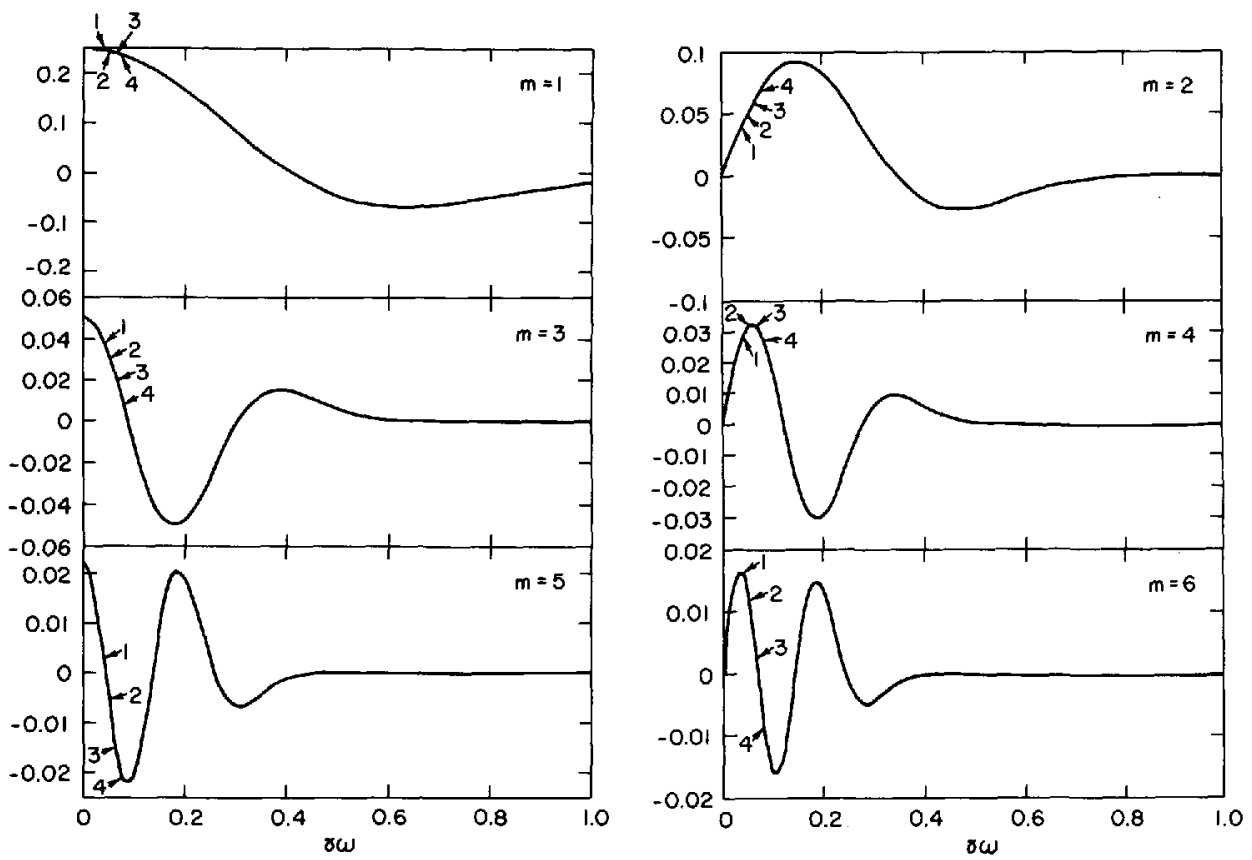

Figure 2. (a) Real part of the amplitude $A_{K}$ of a reflected Kelvin wave generated by incident long Rossby waves for $m$ odd. (b) Imaginary part of the amplitude $A_{K}$ for $m$ even.

A bit of algebra gives the formula for the incident normal mass flux of the $m^{\text {th }}$ Rossby wave of unit amplitude:

$$
U_{m}=\sqrt{2 \pi}(i)^{m+1}\left\{h_{m}[(2 m+1) \gamma \omega]-\gamma \omega \psi_{m}[(2 m+1) \gamma \omega]\right\} .
$$

Note that if $\gamma$ is very small, $O(\omega)$, so the coast is nearly meridional, then the last term can be omitted because the theory is accurate only to $0\left(\omega^{2}\right)$. Importantly, note that all the fluxes depend on slope and frequency only in the combination $\gamma \omega$.

From (4) and (10) it follows that the amplitude of the Kelvin wave reflection of the $m^{\text {th }}$ Rossby wave is

$$
A_{K}=-U_{m}[\gamma \omega] /\left\{\sqrt{\pi} \psi_{0}[\gamma \omega]\right\}
$$

The shapes of $A_{K}$ and $U_{m}$ as functions of $\gamma \omega$ are very similar, differing only by the Gaussian in the denominator of (13). Figure 2a displays $A_{K}$ for $m=1,3,5$ and Figure $2 \mathrm{~b}$ shows it for $m=2,4,6$. For $m$ odd ( $u_{m}$ symmetric about the equator), $U_{m}$ and $A_{K}$ are purely real, while for $m$ even they are purely imaginary. In the latter case they are zero for a meridionally oriented coast. The oscillatory nature of $A_{K}$ could be anticipated from (12). Notice that as $m$ increases $A_{K}$ approaches zero more rapidly with $\gamma \omega$. While the turning points (i.e., the switchover from oscillatory to exponentially decaying behavior) of $h_{m}(y)$ and $\psi_{m}(y)$ occur at $y \simeq(2 m+1)^{1 / 2}$, the arguments of $h_{m}$ and $\psi_{m}$ in 
Table 1. Values for the first six baroclinic modes of the Indian Ocean. The $h_{e}$ 's are the equivalent depths given in Gent et al. (1983), and $\omega$ is the nondimensional semiannual frequency. Taking the angle the African coast makes with the equator to be $50^{\circ}, \gamma=\tan 40^{\circ}$. $X_{E}$ is the nondimensional length of the basin based on a dimensional length of $6000 \mathrm{~km}$.

$\begin{array}{lcccccc}n & 1 & 2 & 3 & 4 & 5 & 6 \\ h_{e}(\mathrm{~cm}) & 80 & 31 & 13 & 7 & 4 & 3 \\ \gamma \omega & .042 & .053 & .066 & .078 & .086 & .096 \\ \omega X_{E} & 0.86 & 1.38 & 2.15 & 3.00 & 3.62 & 4.47\end{array}$

(12) increase as $(2 m+1)$. Hence the location of the turning point for $A_{K}$ decreases as $(2 m+1)^{-1 / 2}$.

Physically, the increase proportional to $(2 m+1)$ is a consequence of the phase variation along the coast associated with shortening zonal wavelength: since $k_{m}=$ $-(2 m+1) \omega$, this effect causes a phase change of $(2 m+1) \omega \gamma$ along the coast in a unit meridional distance.

We have marked the values of $\gamma \omega$ appropriate to the semiannual oscillation for the first four baroclinic modes of the Indian Ocean on Figure 2. (See Table 1.) Accounting for the slant of the coast makes very little difference for $m=1$ but can clearly be significant for $m>1$. For the $m$ even modes it makes $A_{K}$ nonzero: with a slanting coast antisymmetric modes will generate Kelvin waves. We will return to this point in the discussion.

It is of interest to consider how much of the incident energy is reflected in the Kelvin wave and how much goes into the boundary current to ultimately be dissipated. For any wave the energy flux is $c_{g} E$, where $c_{g}$ is the group velocity and $E$ the energy density:

$$
E=\left\langle\int_{-\infty}^{+\infty} \frac{1}{2}\left(u^{2}+v^{2}+h^{2}\right) d y\right\rangle
$$

where $\left\langle\right.$ > denotes the average over a wavelength. For the Kelvin wave the flux is $1 / 4 A_{K}^{2}$, while for the $m^{\text {th }}$ Rossby wave of unit amplitude it is $-[16 m(m+1)]^{-1}$. Hence the ratio of reflected to incident energy flux is $4 m(m+1) A_{K}^{2}$; this is plotted in Figure 3 .

Our method allows $A_{K}$ to be found without calculating the boundary layer flow explicitly, but the boundary current is of some interest in its own right. The longshore transport $\phi(s)$ may be found by using the streamfunction relation (3) in the no normal flow boundary condition (1); i.e.,

$$
\frac{\partial \phi}{\partial s}=-\mathbf{u}^{B} \cdot \mathbf{n}=(u-\gamma v)_{R+K} \cos \theta,
$$

where the subscript $R+K$ denotes the combined Rossby and Kelvin wave contributions. The boundary condition is $\phi\left(Y_{S}\right)=0$; the way $A_{K}$ is determined then insures that $\phi\left(Y_{N}\right)=0$. 

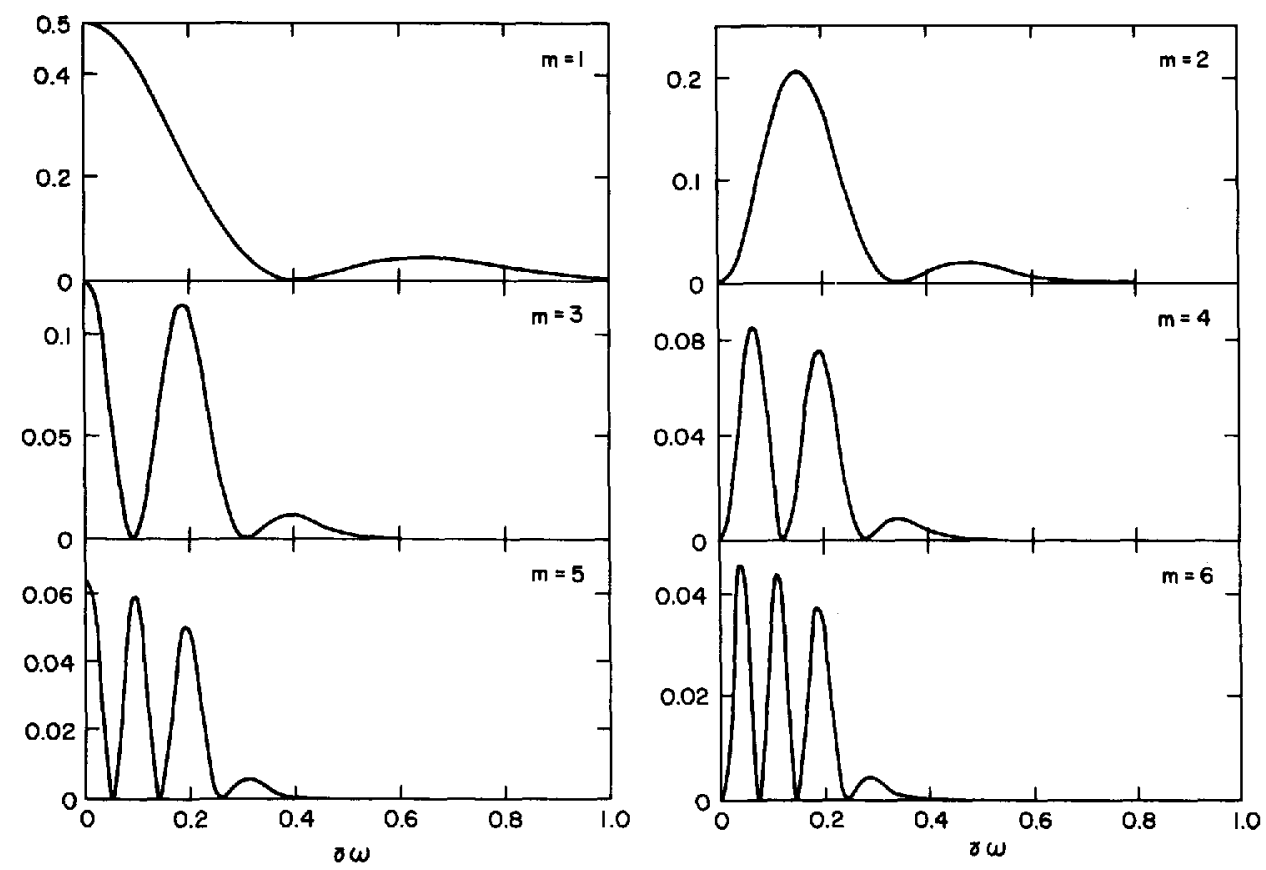

Figure 3. (a) Ratio of energy flux returned in the Kelvin wave to that carried by an incident Rossby wave with $m$ odd. (b) As in (a) for $m$ even.

The longshore transport was calculated numerically for the six gravest Rossby waves with parameters appropriate to the second baroclinic mode semiannual oscillation in the Indian Ocean (see Table 1). Results for the slanting African coast with $\gamma=\tan 40^{\circ}$ are shown in Figures 4 and 5 for $m$ odd and even respectively. The oscillations for each $m$ could be anticipated based on those in the incident Rossby wave zonal velocity. Differences between the meridional coast and the slanting coast increase with increasing $m$ and are greatest at minima of $|\phi|$.

Specific features of the boundary current (e.g., width, velocity profile) depend on the nature of the boundary layer dynamics. For example, if the boundary current is governed by Rayleigh friction with decay time $r^{-1}$ the longshore velocity varies like $\exp \left[-n r \beta /\left(r^{2}+\omega^{2}\right)\right]$, where $n$ is offshore distance. For a semiannual oscillation and a decay time of 2 years the boundary layer scale is $0(400 \mathrm{~km})$.

\section{Results for straight coasts: wind forced solutions}

The results of the previous section show that the effect of a sloping coast varies with meridional wavenumber $m$. The general wind forced response contains all wavenumbers and the net effect will depend on how strongly the different waves contribute to the response. Hence it will depend on the structure of the wind stress forcing. In this section we first show that although the solution for the response must be written as an 

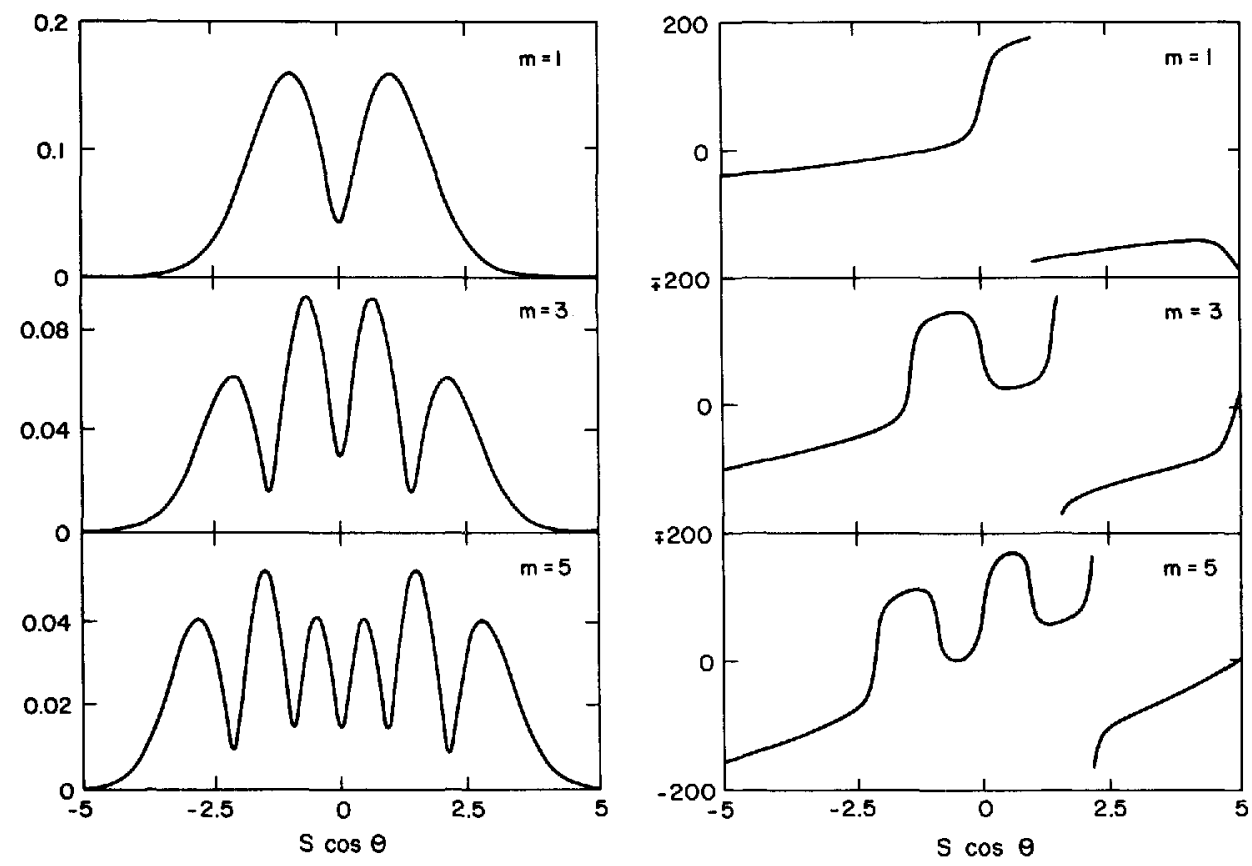

Figure 4. (a) Amplitude and (b) phase of the boundary layer transport $\phi$ generated in response to incident long Rossby waves with $m$ odd. Shown as a function of $y=s \cos \theta$.

infinite sum, the slope of the coast influences only a single complex number $\rho$. We then illustrate the effect of taking $\gamma \neq 0$ for a structurally simple class of forcing functions.

We consider a forced problem in which the forcing is a periodic zonal wind stress with frequency $\omega$. We locate the origin of our coordinate system at the intersection of the western boundary and the equator and take the eastern boundary to be the meridian $x=X_{E}$. The ocean's response may be expressed in the form (cf. CSIV)

$$
(u, v, h)=(i \omega)^{-1} e^{i \omega t}\left\{\left(u_{F}, v_{F}, h_{F}\right)+\rho\left(u_{L}, v_{L}, h_{L}\right)\right\}
$$

where $\left(u_{F}, v_{F}, h_{F}\right)$ is the directly forced part of the solution satisfying $u_{F}=0$ at $x=X_{E}$ and $\left(u_{L}, v_{L}, h_{L}\right)$ is the free mode first discussed by Cane and Moore (1981) consisting of a Kelvin wave and its eastern boundary reflection:

$$
\begin{aligned}
\left(u_{L}, v_{L}, h_{L}\right)= & \left(u_{K}, 0, h_{K}\right) e^{-i \omega\left(x-X_{E}\right)} \\
& +2 \sum_{m=0}^{\infty} \alpha_{m}\left(u_{m}, v_{m}, h_{m}\right) e^{i \omega(2 m+1)\left(x-X_{E}\right)}
\end{aligned}
$$

where $u_{m}$, etc., are given by (11) and the $\alpha_{m}$ are known numbers:

$$
\alpha_{2 m+1}=[(2 m+1) !]^{1 / 2}\left[2^{m} m !\right]^{-1}, \quad \alpha_{2 m}=0 .
$$



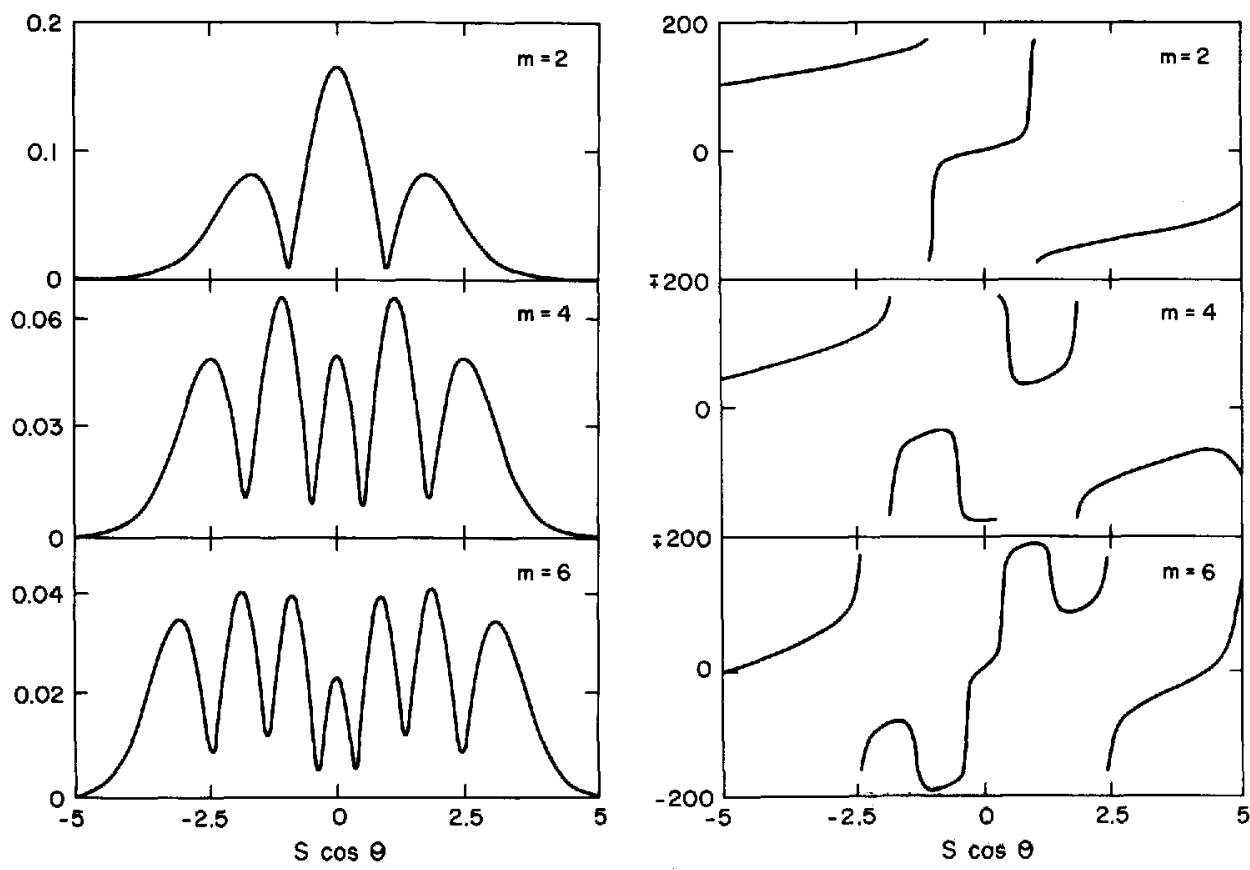

Figure 5. As in Figure 4 but for $m$ even; (a) amplitude, (b) phase.

The Cane and Moore mode is ubiquitous; it is a part of all forced solutions. The amplitude of this free mode, $\rho$, in the response (14), is determined by the western boundary condition that the integral of the mass flux $\mathbf{u} \cdot \mathbf{n}$ vanishes. Letting $U_{L}$ be the integrated mass flux at the western coast due to the velocity $\left(u_{L}, v_{L}\right)$ and denoting the integrated mass flux at the boundary associated with the velocity $\left(u_{F}, v_{F}\right)$ by $U_{F}$, this condition becomes

$$
\rho=-U_{F} / U_{L}
$$

Since both $U_{F}$ and $U_{L}$ depend on the geometry of the western boundary (e.g., on the parameter $\gamma$ for a straight coast), so must $\rho$. In fact, $\rho$ is the only term in the solution (14) where the boundary geometry enters, making it the best single measure of boundary effects.

We readily calculate that

$$
U_{L}\left(\gamma \omega, \omega X_{E}\right)=U_{K}[\gamma \omega] e^{i \omega X_{E}}+2 \sum_{m=0}^{\infty} \alpha_{m} U_{m}[\gamma \omega] e^{-i(2 m+1) \omega X_{E}}
$$

with $U_{m}$ given by (12). Contours of amplitude and phase of $U_{L}$ with the sum calculated up to $m=100$ are shown in Figure 6. Since for $\gamma \neq 0, U_{m} \rightarrow 0$ rapidly as $m$ increases, this sum is close to the infinite one except along $\gamma=0$. If the sum in (18) is taken to infinity then $U_{L}=\pi^{1 / 4}\left(2 i \sin 2 \omega X_{E}\right)^{1 / 2}$ at $\gamma \omega=0$ (see CSIV). Hence the phase there approaches $45^{\circ}$. Since $U_{m}$ is real for $m$ even, it follows from (18) that the phase is $45^{\circ}$ 
a) AMPLITUDE OF $U_{L}$

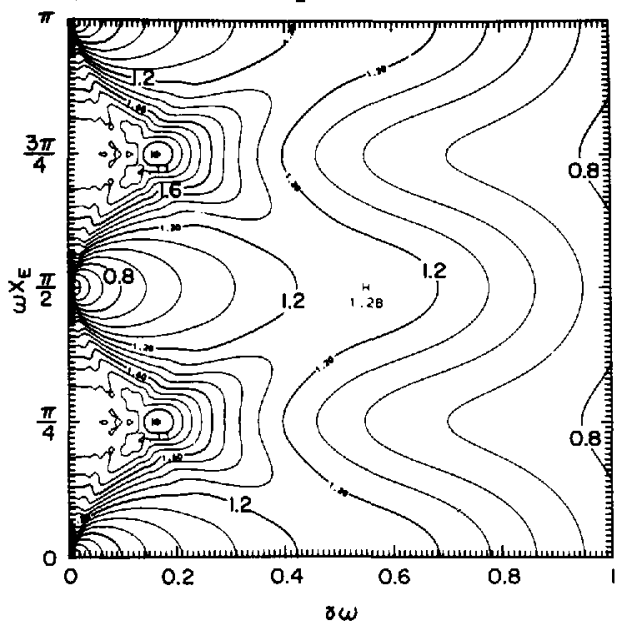

b) PHASE

$C I=15^{\circ}$

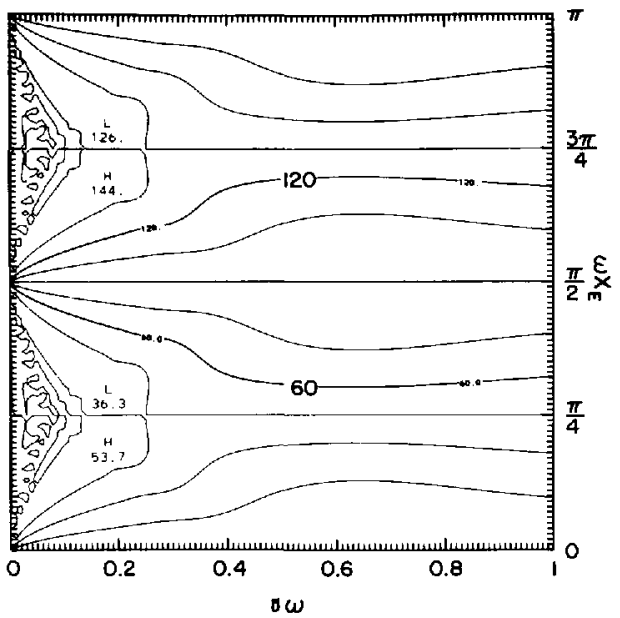

Figure 6. Values of (a) amplitude and (b) phase of $U_{L}$, the western boundary mass flux of the free mode.

for all $\gamma$ when $\omega X_{E}=\pi / 4$. Note that for fixed $\gamma$ the extrema in amplitude lie along the lines $\omega X_{E}=n \pi / 4$, with the overall maximum occurring at $\gamma \omega \simeq .16$. The figure extends only to $\omega X_{E}=\pi$, but $U_{L}\left(-\omega X_{E}\right)=U_{L}^{*}\left(\omega X_{E}\right)$, where "*" denotes complex conjugate.

We now restrict ourselves to zonal wind forcings that are functions of latitude only: $\tau^{(x)}=F(y) e^{i \omega t}$. In this case (CSIV, Eq. (19)),

$$
\begin{aligned}
\left(u_{F}, h_{F}\right)= & d_{K}\left(u_{K}, h_{K}\right)\left[1-e^{-i \omega\left(x-X_{E}\right)}\right] \\
& +\sum_{m=0}^{\infty} r_{m}\left(u_{m}, h_{m}\right)\left[1-e^{i \omega(2 m+1)\left(x-X_{E}\right)}\right]
\end{aligned}
$$

and (cf. CSII, Eq. (9b))

$$
v_{F}=-\sum_{m=0}^{\infty} i \omega\left\{(2 m+1)^{-1} d_{m}+r_{m} e^{i \omega(2 m+1)\left(x-X_{E}\right)}\right\} \psi_{m}(y)
$$

where the $r$ 's and $d$ 's depend on $F$ and $r_{0}=0$. Using the notation

$$
\begin{aligned}
& (f)_{m}=\int_{-\infty}^{+\infty} f(y) \psi_{m}(y) d y, \\
& d_{K}=2^{-1 / 2}(F)_{0} ; \quad d_{m}=(y F)_{m} ; \quad r_{m}=\left(d F / d y-(2 m+1)^{-1} y F\right)_{m} .
\end{aligned}
$$

Direct calculation from (19) yields

$$
\begin{aligned}
-U_{F}\left(\gamma \omega, \omega X_{E}\right)= & d_{K}\left\{U_{K}[\gamma \omega] e^{i \omega X_{E}}-\pi^{1 / 4}\right\} \\
& +\sum_{m=0}^{\infty}\left\{r_{m} U_{m}[\gamma \omega] e^{-i(2 m+1) \omega X_{E}}+\frac{\pi^{1 / 4} \alpha_{m} r_{m}}{2 m(m+1)}\right. \\
& \left.-i \gamma \omega d_{m} \frac{\sqrt{2} \pi^{1 / 4} \alpha_{m+1}}{(2 m+1) \sqrt{m+1}}\right\}
\end{aligned}
$$


a) AMPLITUDE OF $P$

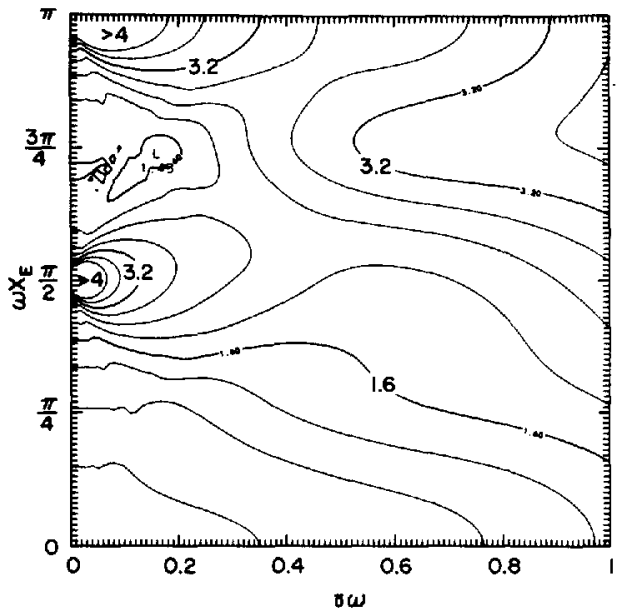

b) PHASE

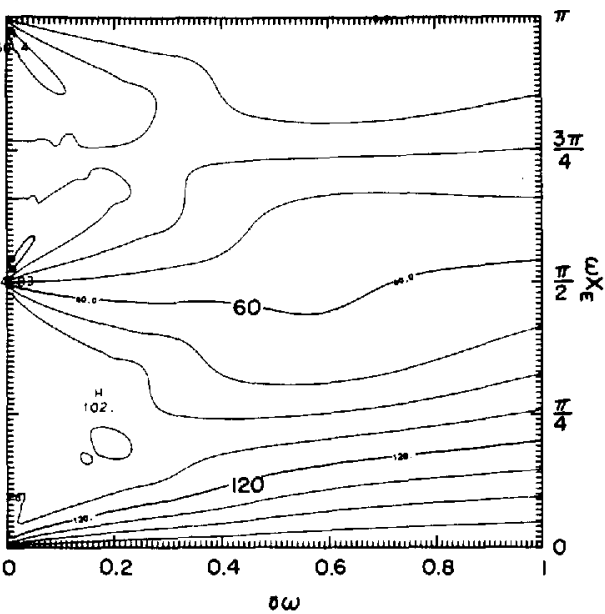

Figure 7. (a) Amplitude and (b) phase of $\rho$ for the symmetric zonal wind forcing $\exp \left(-\mu y^{2} / 2\right)$ for $\mu=0.2$.

This expression simplifies somewhat for purely symmetric or antisymmetric forcing. For the former, $r_{m}=d_{m}=0$ for $m$ even, so the sum may be taken only over $m$ odd. Also, since $\alpha_{m+1}=0$ for $m$ odd the last term vanishes for all $m$. For antisymmetric forcing $d_{k}=0$ and the sum may be taken only over $m$ even; since $\alpha_{m}=0$ for $m$ even, the second term in the sum vanishes.

Figures 7 and 8 show results when the forcing takes the simple symmetric form $F=$

a) AMPLITUDE OF $U$ AT $Y=0$

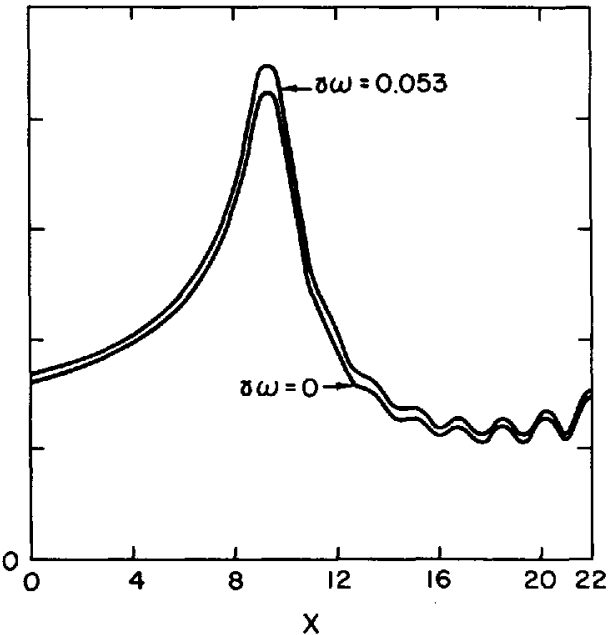

b) AMPLITUDE OF $U$ AT $Y=2$

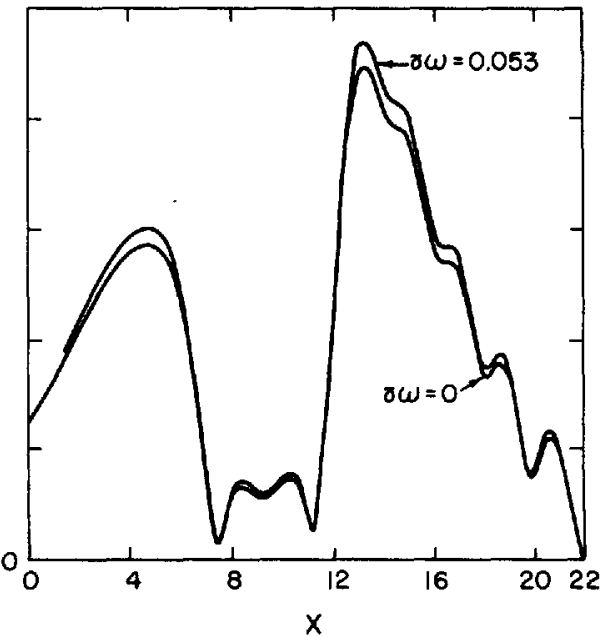

Figure 8. Zonal velocity $u$ for parameters corresponding to the second baroclinic mode semiannual oscillation in the Indian Ocean for the symmetric forcing of Figure 7. Values at $y=0,2$ are shown for $\gamma=\tan 40^{\circ}$ and for $\gamma=0$. 
a) AMPLITUDE OF $P$

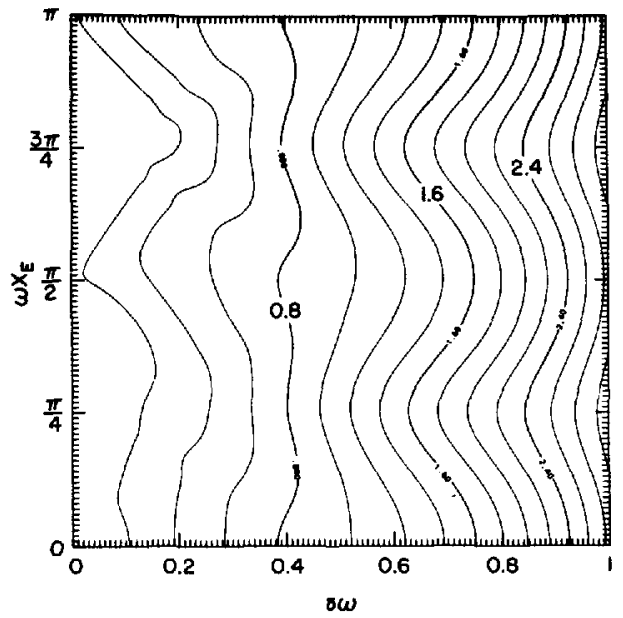

b) PHASE

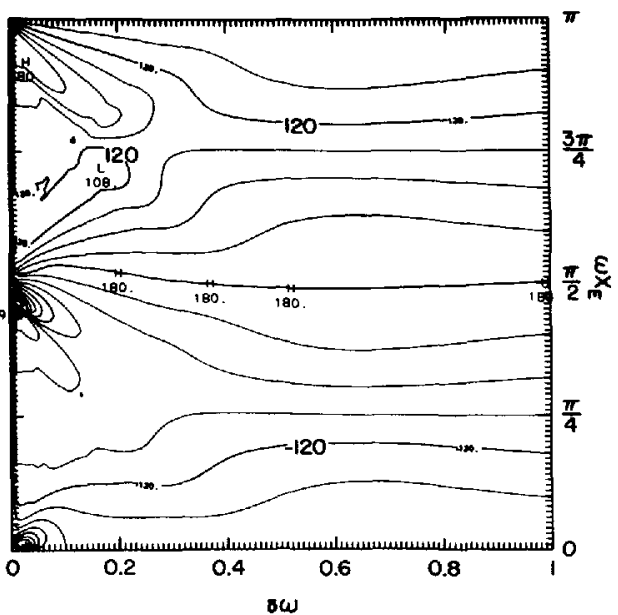

Figure 9. (a) Amplitude and (b) phase of $\rho$ for the antisymmetric zonal wind forcing $y \exp \left(-\mu y^{2} / 2\right)$ for $\mu=0.2$.

$\exp \left(-\mu y^{2} / 2\right)$, which was considered previously in CSIV. Zonal currents and heights along the equator are often indicative of the response to more complex wind stress patterns; cf Gent et al. (1983).

Figure 7 shows the amplitude and phase of $\rho$ with a fairly wide forcing, $\mu=.2$. The figure was drawn with the sums in (18) and (20) taken to $m=100$. [It is shown for $0 \leq$ $\omega X_{E} \leq \pi$; for symmetric forcings with $F(y)$ real, $\rho\left(-\omega X_{E}\right)=\rho^{*}\left(\omega X_{E}\right)$.] Amplitude tends to increase with increasing $\gamma \omega$ and $\omega X_{E}$ because $U_{L}$, the denominator in (17), falls off more rapidly than the numerator, $U_{F}$. In this inviscid case amplitude becomes infinite at the resonance points $\gamma \omega=0, \omega X_{E}=n \pi / 2, n \neq 0$. Phase depends primarily on $\omega X_{E}$, except that for small $\gamma \omega$ it is nearly constant within each $\pi / 2$ interval. Results are not very sensitive to the width of the forcing region once the $e$-folding scale $(2 / \mu)^{1 / 2}$ is greater than one. In general, amplitude increases with increasing scale and phase is nearly constant.

Figure 8 plots values of $u$ for a case corresponding to the second baroclinic mode semiannual oscillation in the Indian Ocean. Parameter values are given in Table 1 . We also include a Rayleigh friction $r=.0644 \omega$, the best case value in Gent et al. (1983). As in that work, the sums are taken to $m=30$; the result is indistinguishable from that for higher $m$ except very close to the eastern boundary. The forcing again has $\mu=.2$, corresponding to an $e$-folding scale of $870 \mathrm{~km}$ for this length scale of $275 \mathrm{~km}$. Amplitudes at $y=0$ and $y=2$ are shown both for a meridional coast $\gamma=0$ and for $\gamma=$ $\tan 40^{\circ}$, appropriate to the African coast. Phases for the two cases are virtually indistinguishable. The slanting coast increases $|\rho|$ by about $8 \%$ because of the decrease in the free mode mass flux, $U_{L}$. In the inviscid case the increase would be about $20 \%$ (see Fig. 7).

Figure 9 shows $\rho$ for the antisymmetric forcing $F=y \exp \left(-\mu y^{2} / 2\right)$ with $\mu=.2$. [To 
a) AMPLITUDE OF U AT $Y=0$

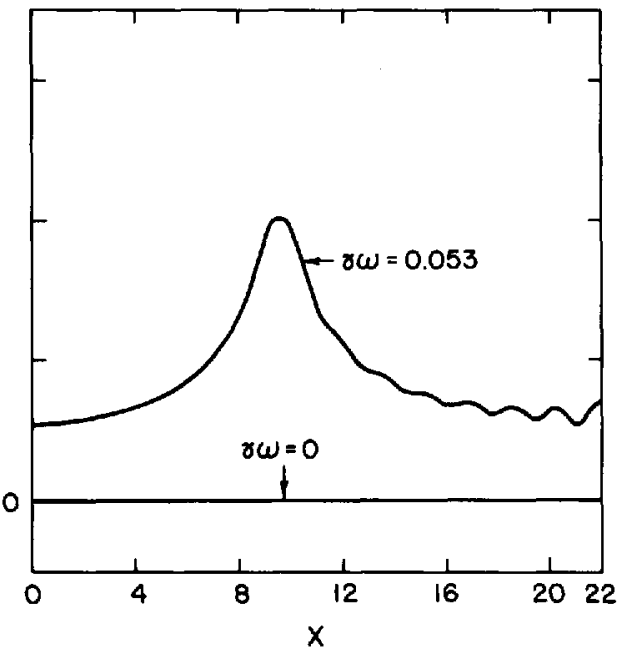

b) AMPLITUDE OF $U$ AT $Y=2$

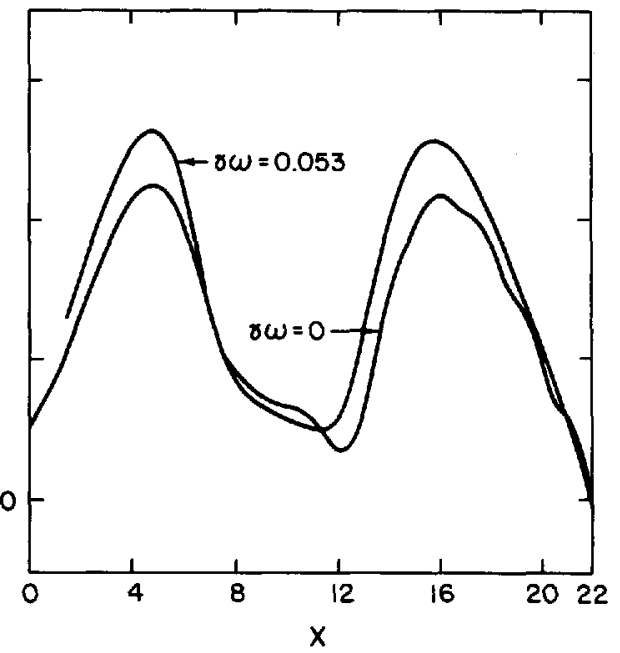

Figure 10. Like Figure 8 but for the antisymmetric forcing $y \exp \left(-\mu y^{2} / 2\right)$.

extend the range of $\omega X_{E}$, note that the antisymmetric forcings with $F(y)$ real, $\rho\left(-\omega X_{E}\right)=-\rho^{*}\left(\omega X_{E}\right)$.] The amplitude is zero for $\gamma=0$ and increases with $\gamma \omega$ more or less independent of $\omega X_{E}$. For $\gamma \omega \gg .1$, when $|\rho|$ is large, the phase decreases with $\omega X_{E}$ nearly independent of $\gamma \omega$. The phase behavior is more complex where $|\rho|$ is small. As the forcing width increases ( $\mu$ decreases), phase stays approximately constant while $|\rho|$ increases sharply for the same values of $\gamma \omega$ and $\omega X_{E}$. Contours of amplitude and phase for larger $\mu$ are similar to Figure 9 except for an increase in the range of $\gamma \omega$ for which $|\rho| \ll 1$ and hence where phase behavior is complicated. Figure 10 has the same parameters as Figure 8 (including Rayleigh friction), except that the forcing is antisymmetric. Again, the phases are very similar for the two coasts except at the equator where $u \equiv 0$ for the straight coast. Obviously, the largest relative change occurs very near the equator, where the straight coast response is small; by $y=2$ the amplitudes are comparable.

\section{Discussion}

We have developed a procedure for calculating effects of western boundaries of arbitrary geometry on low-frequency equatorial waves. This procedure exploits the fact that at low frequencies there is a streamfunction for the sum of eastward propagating short Rossby waves and that the only part of the reflection that propagates away from the boundary is the equatorial Kelvin wave. Formulae for the amplitude of this wave are presented for the case of a uniformly slanted coast when the incident motion is (i) a free Rossby wave and (ii) the result of a zonal wind forcing of a specific type.

In general, the amplitude of the reflected Kelvin wave is determined by the principle 
that its eastward mass flux equals the net mass flux incident at the coast. The latter depends on the slope of the coast because the degree of constructive or destructive interference is influenced by the phase variation along the coast. This effect depends on slope and frequency only in the combination $\gamma \omega$, where $\gamma$ is the slope of the coast and $\omega$ is the (nondimensional) frequency.

Our results for a uniformly slanted coast are most usefully applied to the Indian Ocean. (The western boundaries of the Atlantic and Pacific require numerical integration of (6). Alternately, they can be modeled as a series of discrete steps as in Du Penhoat et al., 1983.) For symmetric motions and forcings (see Figs. 2, 3, 7 and 8) the difference resulting from taking correct account of the slanting coast is not large for the graver baroclinic modes, but there should be notable effects for the higher modes. (For an account of how the various modes influence currents at different longitudes and depths along the equator see Gent et al., 1983.)

However, when comparing with data the changes in the graver modes may prove to be significant. Gent et al.'s (1983) calculated response at the equator has the correct phase, but the amplitude is weak. They also found that the second baroclinic mode made the largest contribution. For the idealized forcing considered here, we found the sloping coast increased the amplitude of the second baroclinic mode currents while leaving the phase unchanged. This increase arose from a change in the free mode mass flux $U_{L}$ rather than the directly forced part of the solution. Therefore, we expect it to be present for more complicated, realistic forcings, improving agreement with observations.

For antisymmetric motions and forcings (see Figs. 2, 3,9 and 10) accounting for the slant of the coast makes a significant difference. Again, the higher the mode (and hence the larger the nondimensional parameter $\gamma \omega$ ) the greater the effect. The antisymmetric component of the zonal wind is not especially large in the Indian Ocean so we expect that it will have little effect on equatorial currents. However, antisymmetric motions are also generated by symmetric meridional winds and the amplitude of such winds in the Somali Jet is very large, larger than any other feature of the equatorial wind field. We therefore conjecture that the combined effect of the Somali Jet and the sloping coast substantially influences Indian Ocean equatorial currents.

Acknowledgments. Valuable discussions with Dr. Kit O'Neill are gratefully acknowledged. M.A.C. was supported by NSF grant OCE-8214771 and NOAA grant NA83RAC00058 to MIT. NCAR is about $80 \%$ sponsored by the National Science Foundation.

\section{REFERENCES}

Cane, M. A. and D. W. Moore. 1981. A note on low frequency equatorial basin modes. J. Phys. Oceanogr., $11,1578-1584$.

Cane, M. A. and E. S. Sarachik. 1977. Forced baroclinic ocean motions. II. The linear equatorial bounded case. J. Mar. Res., 35, 395-432.

— 1979. Forced baroclinic ocean motions. III: The linear equatorial basin case. J. Mar. Res., $37,355-398$. 
1981. The response of a linear baroclinic ocean to periodic forcing. J. Mar. Res., 39, 651-693.

1983. Equatorial oceanography. Rev. Geophys. and Space Phys., 21, 1137-1148.

Du Penhoat, Y., M. A. Cane and R. Patton. 1983. Reflections of low frequency equatorial waves on partial boundaries. Mem. Soc. Royales des Sci. de Liege, J. Nihoul (ed) $6^{e}$ Serie, Tome XIV, 237-258.

Gent, P. R., K. O'Neill and M. A. Cane. 1983. A model of the semiannual oscillation in the equatorial Indian Ocean. J. Phys. Oceanogr., 13, 2148-2160.

Lighthill, M. J. 1969. Dynamic response of the Indian Ocean to onset of the southwest monsoon. Phil. Trans. Roy. Soc. London, A265, 45-92.

Moore, D. W. 1968. Planetary-gravity waves in an equatorial ocean. Ph.D. dissertation, Harvard University, Cambridge, MA.

Moore, D. W. and S. G. H. Philander. 1977. Modeling of the tropical ocean circulation, in The Sea, Vol. 6, Interscience, 319-361. 